\title{
Evaluation of Flue-Gas Desulfurization gypsum in Poultry Litter as a Substrate Component for Greenhouse Horticultural Crops
}

\author{
CJ Paul ${ }^{1}$, CW Robinson ${ }^{1}$, JR Kessler ${ }^{1}$, DE Wells ${ }^{1}$, JL Sibley ${ }^{*}$, HA Torbert ${ }^{2}$ and DB Watts ${ }^{2}$ \\ ${ }^{1}$ Department of Horticulture, Auburn University, USA \\ ${ }^{2}$ USDA-Agricultural Research Service, National Soil Dynamics Laboratory, USA
}

Submission: December 16, 2017; Published: January 22, 2018

"Corresponding author: JL Sibley, Department of Horticulture, Auburn University, AL 36849, USA, Email: sibleje@auburn.edu

\begin{abstract}
A study was conducted to evaluate the growth response and consumer preference of three plant species to substrate blends containing flue gas desulfurization gypsum (FGDG). Substrate blends used in this study were derived from a previous experiment that evaluated the use of FGD Gas a bedding material for broiler chicken production. Five litter treatments chosen from the broiler study were mixed at a 50:50 ratio with crushed pine bark (CPB) giving rise to the following treatments: Pine shavings (PS)+CPB (50:50 v/v); FGDG + PS + CPB(25:25:50 v/v/v); FGDG $+\mathrm{CPB}(50: 50 \mathrm{v} / \mathrm{v})$, Pine bark (PB)+ CPB (50:50 v/v); and FGDG + PB + CPB (25:25:50v/v/v). These treatments were compared to CPB + Farfard 3B $(50: 50 \mathrm{v} / \mathrm{v})$ as the control (industry stand and).The five broiler litter based substrates (treatments) contained poultry manure while the control did not. There were differences in consumer preference, plant growth, foliar greenness (SPAD values), and drainage rates among substrates (treatments) and their suitability for growing plants. In the substrate based on poultry litter from 100\% FGDG bedding, flocculation created good drainage, higher CEC, and greater pore space. This 1: 1 FGDG: CPB substrate had a lower, more desirable pH level, a higher calcium level, and less phosphorus leached from the substrate after watering suggesting the possibility of calcium binding excess phosphorus in the leachate water.
\end{abstract}

Substrates components for greenhouse crops have changed over the years for various reasons. One reason is the availability and costs of substrate components that fluctuate, forcing growers to seek less expensive, readily available alternative substrate components. The primary substrates used in the nursery and greenhouse industry since the 1970's have been pine bark and peat moss. However, in recent years other uses for pine bark have caused a constriction of pine bark availability for horticultural substrates [1]. Increased availability of alternative substrates for the nursery and greenhouse industry has been a justified pursuit for much research [2,3]. Poultry litter has been one of the materials considered for potential use in the nursery industry [4-6], but most of the litter evaluated in the past has been pine wood shavings or sawdust, with very few studies evaluating litter based on pine bark bedding [7]. Gypsum has also been considered as a substrate component for growing horticultural crops $[8,9]$.

An important influence on best practices across a number of agricultural and industrial industries has been federal environmental protection regulations. Two industries, the poultry industry and coal-fired electric companies, are currently required to manage their waste differently than in the past. For example, large poultry operations are required to submit and use a Water and Nutrient Management Plan [10].

There are numerous reasons why alternative substrate options are needed. Costs of materials can become prohibitive, availability may change [2], consumer preferences may change [5,11], and environmental concerns [3,6,8,10,12-16] and regulations Agriculture and Agri-Food Canada [17], may alter recommended best practices [10].

Research seeking alternative substrates increased in the 1970s resulting from increased populations with an increased demand for container grown plants nationwide [18], erratic supplies of peat moss, and a need to reduce landfill use [3-5,13,14,17,19-21]. Horticulturists are in a unique position to help solve pollution problems caused by the disposal of certain waste materials, which would otherwise become environmental problems $[3,13,16,22]$.

Some materials evaluated for use as horticultural substrate components have included spent tea grinds [23], gasifier residue [24], clean chip residual and processed whole pine trees [2]. Results from studies using composted chicken litter as an alternative to inorganic fertilizers in the landscape and as a substrate component for containers proved suitable in both cases [6]. In another study bio-solids saturated newspaper crumbles or composted poultry litter was added at $25 \%$ vol:vol to either ground pine chips or pine bark. Substrates amended with composted poultry litter produced the largest plants across all treatments [3]. Gypsum has been studied both as a substrate component as well as a fertilizer and chemical stabilizer of phosphorus in poultry litter, with the intent of minimizing negative environmental impact on local ground water and waterways with great success $[8,16,25,22]$.

Keywords: Agricultural wastes; Industrial wastes; Re-use; Alternative potting media 


\section{The Poultry Industry}

The poultry industry is the largest agricultural industry in however, a problem with poultry production is what to do with the waste produced, primarily from processing plant waste and bedding material or poultry litter. Processing plant waste is generally cooked for use in pet food Sibley 2016 however utilization of the bedding is still an issue for the industry [3$5,13,17,20,21,22,26]$.

In recent years, a growing need for alternative methods of managing wastes for poultry production coupled with increased need for alternative components for use in horticulture industry substrates was the focus of much research [2,3,6,10,16,19,22,27]. There is good reason for this interest in planned manure management that includes re-use. Generally, animals only use about $25 \%$ of the nutrients they receive in their feed with manure trapping about $75 \%$ of the nutrients in the original feed $(\mathrm{N}, \mathrm{P}, \mathrm{K})$, nutrients that are potentially useful to crops and plants.

Two methods of composting poultry litter (PL), windrow and in-vessel digester, with the potential for use in the green industry were compared by Brymer [19]. Data indicated electrical conductivity and $\mathrm{pH}$ levels for in-vessel derived compost might not be suitable for production without pre-plant leaching when growing salt and $\mathrm{pH}$ sensitive plants. Burning poultry litter for energy production and using the poultry litter ash in greenhouse crop production, specifically as an alternative fertilizer, was successful in Louisiana [22]. The burning process changed the phosphorus (P) in the poultry litter from a soluble form (that is readily available) to strong bonds of $\mathrm{P}$, specifically tricalcium phosphate. The heat burned off the organic materials like nitrogen and carbon, therefore, only minerals remained, with the minerals (in this case $\mathrm{Ca}$ and $\mathrm{P}$ ) forming tight bonds with each other. The substrates with poultry litter ash (PLA) incorporated leached only 10 to $20 \mathrm{ppm} \mathrm{P}$, unlike monocalcium phosphate with 2,000ppm P solubility, or dicalcium phosphate with 200ppm P. This reduced phosphorus runoff from greenhouse-grown plants, but still provided the phosphorus required for healthy growth [22].

The poultry industry is interested in bedding materials that are inexpensive, available in large quantities without excessive shipping costs, and that take up and release moisture without damaging effects to bird health or product quality. Numerous materials have been considered for poultry bedding [28,29]. In one study, pododermatitis was generally low in pine shavings, pine bark, sand and gypsum [29]. Bird mortality rates were lowest with gypsum bedding and birds had higher body weights and feed efficiency with gypsum bedding [29].

Poultry litter (PL) has been used as a substrate, a soil conditioner, and fertilizer, but given the quantity of PL produced in some states like Alabama and Georgia, excess PL creates challenges in waste disposal and concerns about pollution. Pollution can come from erosion of amended soils, farm pasture runoff, leachates coming from nursey, or greenhouse containers that have PL as a substrate component. Leachates or runoff often contain highly soluble phosphorus and other nutrients that pose pollution concerns if they are able to enter local waterways, potentially causing algae blooms, which can use up the oxygen in bodies of water creating fish kills and making the water unfit for human consumption $[3,6,13,14,20,21]$.

In a study evaluating PL in amended raised landscape beds with cotton gin waste and/or pine bark, cotton gin waste removed the odor from the PL, and the composted waste material performed equally to the standard peat moss [5]. Also in 1991, bagged soilless potting mix amended with composted PL was acceptable to consumers [4].

Use of gypsum as a way of treating PL to reduce the concentration of P loss from PL amended soils and substrates was evaluated. Gypsum was applied at four rates with the concentration of soluble reactive phosphorus (SRP) significantly reduced even at the lowest gypsum application rate compared with no gypsum application at all sampling periods [16].

\section{Flue Gas De-sulfurization Gypsum}

Gypsum has been used in the United States for over 250 years as a fertilizer to provide the essential nutrients calcium and sulfur and to improve overall plant growth. Gypsum added to the soil can also improve the chemical and physical properties of some soils (especially heavy clay soils- causing flocculation of the soil and creating aggregates), and thus can reduce surface crust formation and increase water infiltration rates and movement of water and gases through the soil profile, ultimately reducing erosion and nutrient losses (especially of phosphorus) in surface water runoff. Chemical properties improved by application of gypsum include the mitigation of subsoil acidity and aluminum toxicity, while enhancing deeper root growth and the ability of plants to take up water and nutrients, especially during drought periods. Gypsum is the most common amendment for sodic soil reclamation and can be included as a component in synthetic soils for nursery, greenhouse and landscape uses [8].

There are several sources of gypsum available for agricultural use in the United States. These include the traditional gypsum that is mined, reclaimed casting gypsum from industry, recycled wallboard and flue gas desulfurization gypsum (FGDG) from power plants. FGDG is a somewhat newer source that produces a large volume of gypsum that, as of today, does not have clear re-use solutions. Instead, large quantities of gypsum are being placed in landfills, deposited in surface impoundments, or beneficially recycled [8].

In 2001, combustion of coal was producing $52 \%$ of the electricity in the U.S. [8]. Because of the Clean Air Act of 1970 (amended in 1990), coal fired electric plants were mandated to capture all sulfur dioxide $\left(\mathrm{SO}_{2}\right)$ emissions and other components of air pollution from burning coal by the year 2010. To do this, the utility companies installed SO2 scrubbers, which use calcium carbonate or hydrated lime $\left(\mathrm{CaCO}_{3}\right)$ in a slurry form, to filter fumes as they are passed through. This process converts the slurry into calcium sulfate (gypsum - $\mathrm{CaSO}_{4} \cdot 2 \mathrm{H}_{2} \mathrm{O}$ ) and $\mathrm{CO}_{2}$ 
which is not considered a pollutant. As a result, since 1970, every coal-fired plant has either closed or been outfitted with scrubbers.

The good news is, FGD Gis high quality, suitable for agricultural use, and qualifies as a recycling process itself [30]. The bad news is that current distribution channels of gypsum are unable to utilize the quantities of the new gypsum supplies produced from flue gas desulfurization filters. In 2008, approximately 18 million tons of FGDG were produced, of which $60 \%$ (10.6 million tons) was used (mainly in wallboard) with less than $2 \%$ used in agriculture. Because it is well known that mined gypsum can improve soil structure and help prevent pollution of local waterways, there is great interest in using the high-quality FGDG produced by utilities to replace mined gypsum [8]. In 2011, the Ohio State University Cooperative Extension Service created a comprehensive bulletin to inform the public and industry about the potential agricultural uses of gypsum. Topics include everything from reviewing properties of gypsum, to benefits for agriculture, application uses, economic considerations, analytical and technical support, and gypsum handling and storage [8].

Because of the increased availability of FGDG, in 2013, the USDA-ARS ran a FGDG project at several locations including Mississippi, Tennessee, Georgia, and Alabama to look at the potential value of FGDG as a soil amendment in agriculture [25]. Further research investigated the impact of FGDG application on water quality in a coastal plain area in combination with PL used in field application [16].

The objective of this study was to evaluate different pine bark and pine shaving litter blends with or without gypsum used as a substrate material for greenhouse horticultural crops.

\section{Materials and Methods}

Substrate used for this study came from a previous experiment watts et al. 2017 evaluated the influence of different bedding materials on broiler chicken production after rearing three flocks. Five poultry litter (PL) treatments (PL; used bedding + broiler manure) chosen from the broiler study were mixed at a 50:50 ratio with crushed pine bark (CPB) giving rise to the following treatments: $100 \%$ Pine shavings (PS) + CPB(50:50v/v); 50\% FGDG + 50\% PS + CPB (25:25:50v/v/v); $100 \%$ FGDG + CPB (50:50v/v), 100\% Pine bark (PB) + CPB $(50: 50 \mathrm{v} / \mathrm{v})$; and 50\% FGDG + 50\% PB+CPB $(25: 25: 50 \mathrm{v} / \mathrm{v} / \mathrm{v})$. These treatments were compared to CPB + Farfard 3B (50:50v/v) as the control (industry stand and). Each of these PL treatments were mixed with crushed pine bark (CPB) at a 50:50 ratio and compared to a control composed of CPB (3/8" sieve) + Fafard 3B (50:50). Thus, the resultant substrate treatments used were: $A$ - PS+CPB (50:50v/v), B - FGDG+PS+CPB (25:25:50v/v/v); C $\mathrm{FGDG}+\mathrm{CPB}(50 \mathrm{v} / \mathrm{v} / \mathrm{v}), \mathrm{D}-\mathrm{PB}+\mathrm{CPB}(50: 50 \mathrm{v} / \mathrm{v}), \mathrm{E}-\mathrm{FGDG}+\mathrm{PB}+\mathrm{CPB}$ (25:25:50v/v/v), and control - CPB+Farfard3B (50:50v/v). All five of the treatments derived from the broiler study contained manure, while the control treatment did not. For the control, Fafard 3B was substituted for the broiler manure. The substrates (treatments) were combined by mixing one cubic foot $\left(0.03 \mathrm{~m}^{3}\right)$ of each PL treatment with one cubic foot of CPB, thoroughly mixed using an automatic mixing machine before use with no additional amendments.

The experiment was initiated on August 19, 2016in a $2300 \mathrm{ft}^{2}$ fan and padat the Paterson Greenhouse Complex, Auburn University, AL. Pots (5" x 6.5”) (12.7 x $16.5 \mathrm{~cm})$ were loosely filled with substrate and shaken to level. Australian sword fern (Nephrolepis obliterata $R$. Br.), Japanese painted fern (Athyriumniponicum var. Pictum Mett.), and Gerbera daisy (Gerbera jamesonii L.'Revolution Yellow') were transplanted from either 50- or 72-cell pack into the pots (Gerbera daisy 50-cell packs; both ferns 72-cell packs (Casa Flora, Texas)) containing the different substrates. Each substrate treatment was subjected to a fertilized vs. non-fertilized regimen [31]. After transplanting, each treatment was arranged by species and fertilizer regimen in a randomized complete block design. Both fern species had 10 single pot replications $x 2$ fertilization regimens for each treatment. The Gerbera daisy had 8 single pot replications $\mathrm{x} 2$ fertilization regimens for each treatment. Thus, there were a total of 336 plants for this experiment, all of which were grown for 50 days. All plants were irrigated daily and fertilized plants received 150ppm N solution (beginning $2 \mathrm{wk}$ after transplanting) through the irrigation system. The irrigation water had an average $\mathrm{pH}$ of 7.0 during this study.

Before substrates were mixed with the CPB, a sample was taken from each treatment and submitted to Auburn University Soil Testing Laboratory (AUSTL) for analysis of pH, EC, SS, $\mathrm{NO}_{3}-\mathrm{N}, \mathrm{Ca}, \mathrm{K}, \mathrm{Mg}$, and $\mathrm{P}$, plus micronutrients. Bulk density was determined on a total of three pots of dry substrate per treatment including the control prior to and after combination with the CPB. Substrates were leached 10 times before planting and then leachates were collected weekly from four pots per treatment until terminating the experiment using the Virginia Tech Pour Thru Method [32]. Growth indices (in cm) ((height + widest width + width perpendicular to the first)/3) were measured 14,31, and 50 days after transplanting (DAT). Leaf chlorophyll content was quantified using a SPAD-502 chlorophyll meter (Minolta Camera Co., Ramsey, NJ) at 31 and 50 DAT for Australian sword fern and Gerbera daisies. General health and growth vigor was assessed the day of termination (50 DAT) using a scale varying from 1 to 7 depending on the species, where 1 was assigned to plants with the poorest growth and color, and 7 was assigned to plants with the greatest growth, vigor and healthy color. Consumer preference was evaluated by use of a survey. Twelve plants were selected from each species, (one plant from each substrate and fertilizer combination), and were presented to the public with instructions to choose at least one or not more than three plants from each species that they would consider purchasing at a retail location (or none if they would not purchase). The survey ran for 3 days and had 46 participants. An analysis of variance was performed on all responses using PROC GLIMMIX in SAS version 9.4 (SAS Institute, Cary, NC). The Gaussian distribution was used for all responses except consumer preferences where the multinomial 
distribution was used. Where residual plots and a significant covariance test for homogeneity indicated heterogeneous variance among treatments, a RANDOM statement with the GROUP option was used to correct heterogeneity in Gaussian data. Least square means comparisons among treatments and comparisons of treatments to the control were determined using the simulated method. Least squares mean group comparisons of all FDG versus no FDG and high FDG versus low FDG were determined using the simulated method. Differences among treatments of consumer preferences were estimated. All significances were at $\alpha=0.05[33,34]$.

\section{Results}

\section{Consumer preference}

Production of marketable plants is the real verification of suitable cultural practices, therefore this discussion begins with the consumer preference results and relates data collected about the plants grown in substrates with and without gypsum to the ratings.

In general, the survey revealed that FGDG and fertilizer were important substrate components in producing ferns the survey participants said they would purchase. For both fern species, treatment "C", (the substrate that began as 100\% FGDG in the broiler study), was one of the top two plants chosen for potential purchase in the survey (Tables 1-4). Treatment "C" for the Australian sword ferns received 31 "yes" votes (out of 121). Treatment "C" for the Japanese painted ferns received 38 "yes" votes (out of 94). The other top substrate for the Australian sword ferns was treatment "D" (which began as 100\% pine bark in the broiler study), which received 36 "yes" votes (out of 121). The Japanese painted fern in the control treatment consisting of 1:1CPB + Fafard 3B potting mix received 37 "yes" votes (out of 94). The top two treatments for each fern species were statistically equal. The two substrates other than the gypsum, were pine bark based, either $50 \%$ or $100 \%$ which currently is an industry standard. Among the 94 "yes" votes for Japanese painted ferns, the top two rated far above any other substrates. The Australian sword fern had 19 votes given to treatment "E" (50:50 FGDG: PB from the broiler study) and 11 "yes" votes for treatment "B" (50:50 PS:FGDG from the broiler study) out of the 121 "yes" votes. A possible reason for the success of the plants could be that both "B" and "E" treatments contained gypsum as well, (as did treatment "C" that began as $100 \%$ gypsum and was a top vote getter) (Tables 1-4).

Table 1: Consumer preferences and leachate readings at termination for Australian Sword in six substrates.

\begin{tabular}{|c|c|c|c|c|c|c|c|c|c|c|}
\hline \multirow[b]{3}{*}{ Substrate $^{t}$} & \multirow{3}{*}{$\begin{array}{c}\begin{array}{c}\text { Consumer } \\
\text { Preference }^{\mathrm{z}}\end{array} \\
\text { Fertilized } \\
\text { No }\end{array}$} & \multirow[b]{3}{*}{ Yes } & \multirow{3}{*}{$\begin{array}{c}\text { Rating } \\
\text { Substrate }\end{array}$} & \multirow{3}{*}{$\begin{array}{c}\text { Dry } \\
\text { Weight }^{\mathrm{x}} \\
\text { Substrate }\end{array}$} & \multirow{3}{*}{$\begin{array}{c}\text { Hieght }^{w} \\
\text { Fertilized } \\
\text { Yes }\end{array}$} & \multicolumn{2}{|c|}{ Spad $^{v}$} & \multirow{2}{*}{\multicolumn{3}{|c|}{$\begin{array}{c}\text { Size }^{u} \\
\text { rtilize } \mathrm{x} \text { days }\end{array}$}} \\
\hline & & & & & & \multirow{2}{*}{$\begin{array}{c}\text { Fertilized } \\
\text { No }\end{array}$} & \multirow[b]{2}{*}{ yes } & & & \\
\hline & & & & & & & & & & \\
\hline A & $2 d$ & $5 c d^{s}$ & $4.0 \mathrm{abc}$ & $4.0 \mathrm{ab}$ & $14.1 \mathrm{~ns}$ & $35.1 \mathrm{abB}^{*}$ & $47.9 \mathrm{nsA}$ & & & Fertiliszed \\
\hline $\mathrm{B}$ & & $11 \mathrm{bc}$ & $5.0 \mathrm{a} * \mathrm{r}$ & $4.5 \mathrm{ab}$ & $14.9 \mathrm{~A}$ & $38.5 \mathrm{aB}^{*}$ & $49.6 \mathrm{~A}$ & Days & No & Yes \\
\hline $\mathrm{C}$ & $1 \mathrm{~d}$ & $31 \mathrm{a}$ & $4.0 \mathrm{bc}$ & $3.7 \mathrm{~b}$ & 14.5 & $26.9 \mathrm{bcB}$ & $44.5 \mathrm{~A}$ & 14 & 18.8ns & 18.9 \\
\hline $\mathrm{D}$ & $8 \mathrm{~cd}$ & $36 a$ & $5.0 \mathrm{a}$ & $5.3 \mathrm{a}$ & 14.1 & $31.5 a b c B^{*}$ & $51.8 \mathrm{~A}$ & 31 & $18.9 \mathrm{~b}$ & $20.1 \mathrm{a}$ \\
\hline $\mathrm{E}$ & $19 b$ & $0 \mathrm{~d}$ & $5.0 \mathrm{ab}^{*}$ & $3.5 b$ & 14.1 & $23.9 \mathrm{cB}$ & $46.2 \mathrm{~A}$ & 50 & $20.4 b$ & $25.0 \mathrm{a}$ \\
\hline Control & $2 d$ & $6 \mathrm{~cd}$ & $3.5 c$ & $3.4 \mathrm{~b}$ & $14.8 \mathrm{~A}$ & $23.9 \mathrm{cB}$ & $49.8 \mathrm{~A}$ & sign. ${ }^{q}$ & $\mathrm{~L}^{* *}$ & $\mathrm{Q}^{* *}$ \\
\hline \multicolumn{11}{|c|}{ With Fertilizer } \\
\hline No gyspum & & & 4 & 4.6 & 14.1 & & 49.8 & & & \\
\hline $\begin{array}{c}50 \% \\
\text { gysum }\end{array}$ & & & $4.5 \mathrm{~ns}$ & $4.0 \mathrm{~ns}$ & $14.5 \mathrm{~ns}$ & & $47.9 \mathrm{~ns}$ & & & \\
\hline $\begin{array}{c}100 \% \\
\text { gypsum }\end{array}$ & & & 4 & 3.7 & $14.5 \mathrm{~ns}$ & & 44.5 & & & \\
\hline \multicolumn{11}{|c|}{ Fertilizer } \\
\hline No & & & $3.0 \mathrm{~b}$ & $3.0 \mathrm{~b}$ & & & & & & \\
\hline Yes & & & $5.0 \mathrm{a}$ & $5.4 \mathrm{a}$ & & & & & & \\
\hline
\end{tabular}

zThe consumer preference Survey had 41 participants

yReported are medians for rating

$x$ Only the substrate and fertilizer main effects were significant at $a=0.05$.

wThe Substrate by fertilizer Interaction was significant at $\alpha=0.05$.

vOnly the substrate by fertilizer interaction and the days after planting main effect were significant at $\alpha=0.05$.

$t A=1: 1$ Pine Shhavings/crushed pine dark (and chicken manure), $B=1: 1 P S / G y p s u m$ to $2 C P B+C M, C=1: 1 \quad G y p s u m / C P B+C M$, 


\section{Agricultural Research \& Technology: Open Access Journal}

\section{$\mathrm{D}=1: 1 \mathrm{~PB} / \mathrm{CPB}+\mathrm{CM}, \mathrm{E}=1: 1$ Gypsum/PB to $2 \mathrm{CPB}+\mathrm{CM}$, Control= 1:1 Crushed Pine Bark/Fafard 3B Plotting Mix.}

SLeast Squares means comparisions between substrates (Lower acse inrows) using the stimulated method at $s=0.05$, ns $=$ not significant.

rLeast Squares means followed by an asterisk were significantly different from the control using the stimulated method at $\alpha=0.05$.

Table 2: Consumer preferences ${ }^{z}$ and leachate readings ${ }^{y}$ at termination of Australian Sword ferns grown in six substrates . $^{x}$

\begin{tabular}{|c|c|c|c|c|c|c|c|c|c|}
\hline & $\begin{array}{c}\text { Consumer } \\
\text { Preferences }^{\mathrm{z}}\end{array}$ & & $\mathbf{p H}^{\mathbf{y}}$ & & $E^{x}$ & & Ca ppm ${ }^{w}$ & $\mathbf{P} \mathbf{p p m}^{\mathbf{v}}$ & \\
\hline & Fertilized & & Fertilized & & Fertilized & & \multirow{2}{*}{$\begin{array}{l}\text { Fertilzer } x \\
\text { Substrate } \\
\quad=\text { Not } \\
\text { Significant }\end{array}$} & Fertilized & \\
\hline Substrate $^{u}$ & No & Yes & No & Yes & No & Yes & & No & Yes \\
\hline A & $2 \mathrm{~d}$ & $5 \mathrm{cdt}$ & $7.50 \mathrm{aA} * \mathrm{~s}$ & 6.62abB & $0.42 \mathrm{bB}$ & $0.92 \mathrm{cA}$ & $82.0 \mathrm{bc} *$ & $43.4 \mathrm{aB}^{*}$ & $109.4 \mathrm{aA}^{*}$ \\
\hline B & & $11 \mathrm{bc}$ & 7.40abNS & $7.16 a^{*}$ & $2.08 \mathrm{aB} *$ & $2.45 \mathrm{abA}^{*}$ & $564.3 a^{*}$ & 9.7bNS* & $14.1 \mathrm{cff}$ \\
\hline $\mathrm{C}$ & $1 \mathrm{~d}$ & $31 \mathrm{a}$ & $6.56 \mathrm{cNS}$ & $6.49 \mathrm{ab}$ & 2.08aNS* & $2.14 \mathrm{~b}^{*}$ & $581.5 a^{*}$ & $16.9 \mathrm{bB} *$ & $36.8 \mathrm{bA}$ \\
\hline $\mathrm{D}$ & $8 \mathrm{~cd}$ & $36 a$ & $7.26 \mathrm{abA}$ & $6.02 \mathrm{bcB}$ & $0.47 \mathrm{bB}$ & $1.04 \mathrm{cA}$ & $105.2 b^{*}$ & $60.5 \mathrm{aB}^{*}$ & $135.8 \mathrm{a} A^{*}$ \\
\hline $\mathrm{E}$ & $19 b$ & $0 \mathrm{~d}$ & $7.52 \mathrm{aA}^{*}$ & $7.02 \mathrm{aB}$ & $2.20 \mathrm{aB}^{*}$ & $2.54 \mathrm{aA}^{*}$ & $623.0 a^{*}$ & 8.3bNS* & $29.6 b$ \\
\hline Control & $2 \mathrm{~d}$ & $6 \mathrm{~cd}$ & $7.07 \mathrm{bA}$ & $5.63 \mathrm{cB}$ & $0.21 \mathrm{bB}$ & $0.79 \mathrm{cA}$ & $28.2 \mathrm{c}$ & $0.8 \mathrm{cB}$ & $45.8 \mathrm{bA}$ \\
\hline \multicolumn{10}{|c|}{ With fertilizer } \\
\hline $\begin{array}{c}\text { All } \\
\text { gypsum }\end{array}$ & & & & $6.89 a^{r}$ & & $2.4 \mathrm{a}$ & & $589.6 a$ & $26.8 \mathrm{~b}$ \\
\hline $\begin{array}{c}\text { No } \\
\text { gypsum }\end{array}$ & & & & $6.32 \mathrm{~b}$ & & $1.0 \mathrm{~b}$ & & $93.6 \mathrm{~b}$ & $122.6 \mathrm{a}$ \\
\hline $\begin{array}{c}50 \% \\
\text { gypsum }\end{array}$ & & & & $7.09 a$ & & $2.5 \mathrm{a}$ & & $593.7 \mathrm{~ns}$ & $21.8 \mathrm{~b}$ \\
\hline $\begin{array}{c}100 \% \\
\text { gypsum }\end{array}$ & & & & $6.49 \mathrm{~b}$ & & $2.1 \mathrm{~b}$ & & 581.5 & 36.8 a \\
\hline \multicolumn{10}{|c|}{ zThe Consumer Preferences Survey had 46 participants. } \\
\hline \multicolumn{10}{|c|}{ yThe substrate by fertilizer interaction was significant at $\alpha=0.05$. } \\
\hline \multicolumn{10}{|c|}{ xThe substrate by fertilizer interaction was significant at $\alpha=0.05$. } \\
\hline \multicolumn{10}{|c|}{ wOnly the substrate and fertiizer main effects were significant at a=0.05. } \\
\hline \multicolumn{10}{|c|}{ vThe substrate by fertilizer interaction was significant at $\alpha=0.05$. } \\
\hline \multicolumn{10}{|c|}{ uSubstrates: $A=1: 1$ Pine shavings/crushed pine bark (and chicken manure), $B=1: 1 \mathrm{PS} /$ Gypsum to $2 \mathrm{CPB}+\mathrm{CM}, \mathrm{C}=1: 1 \mathrm{Gypsum} / \mathrm{CPB}+\mathrm{CM}, \mathrm{D}=1: 1 \mathrm{~PB} / \mathrm{CPB}+\mathrm{CM}$, } \\
\hline \multicolumn{10}{|c|}{ E=1:1 Gypsum/PB to 2 CPB + CM, Control=1:1 Crushed Pine Bark/Fafard B Potting Mix. } \\
\hline \multicolumn{10}{|c|}{ tLeast squares means comparisons between fertilizers (lower case in rows) using the simulated method at $\alpha=0.05$, ns=not significant. } \\
\hline \multicolumn{10}{|c|}{ sLeast squares means followed by an asterisk were significantly different from the control using the simulated method at $\alpha=0.05$. } \\
\hline
\end{tabular}

Table 3: Consumer preferences and leachate readings at termination of Japanese Painted ferns grown in six substrates.

\begin{tabular}{|c|c|c|c|c|c|c|c|c|c|c|c|c|c|c|c|c|}
\hline \multirow{4}{*}{$\frac{\text { Substrate }}{\text { A }}$} & \multirow{2}{*}{\multicolumn{2}{|c|}{$\begin{array}{c}\begin{array}{c}\text { Consumer } \\
\text { Preferencez }\end{array} \\
\text { Fertilized }\end{array}$}} & \multirow{2}{*}{\multicolumn{2}{|c|}{$\begin{array}{c}\text { Ratingy } \\
\text { Fertilized }\end{array}$}} & \multirow{2}{*}{\multicolumn{2}{|c|}{$\begin{array}{c}\text { Dry Weightx } \\
\text { Fertilized }\end{array}$}} & \multicolumn{4}{|c|}{ Heightw } & \multicolumn{6}{|c|}{ Sizev } \\
\hline & & & & & & & \multicolumn{3}{|c|}{ Fertilized } & \multicolumn{3}{|c|}{ Fertilized } & \multirow{2}{*}{\multicolumn{4}{|c|}{ Fertilizer x Days }} \\
\hline & \multirow{2}{*}{$\frac{\text { No }}{1 \mathrm{nsB}}$} & \multirow{2}{*}{$\frac{\text { Yes }}{5 \mathrm{bA}}$} & \multirow{2}{*}{$\frac{\text { No }}{3.0 \mathrm{nsNS}}$} & \multirow{2}{*}{$\frac{\text { Yes }}{3.0 c^{*} \mathrm{~s}}$} & \multirow{2}{*}{$\frac{\text { No }}{1.2 \mathrm{aNS}^{*}}$} & \multirow{2}{*}{$\begin{array}{c}\text { Yes } \\
0.9 \mathrm{~ns}\end{array}$} & \multirow{2}{*}{$\begin{array}{c}\text { No } \\
14.7 \mathrm{aA}\end{array}$} & \multirow{2}{*}{$\frac{\text { Yes }}{12.5 \mathrm{abB}}$} & \multicolumn{2}{|c|}{ Fertilizer/Height } & \multirow{2}{*}{$\frac{\text { No }}{16.2 \mathrm{aNS}^{*}}$} & \multirow{2}{*}{$\begin{array}{c}\text { Yes } \\
15.7 \mathrm{ab}\end{array}$} & & & & \\
\hline & & & & & & & & & & & & & & & Fertilized & \\
\hline B & $1 \mathrm{nsB}$ & $1 b c$ & $3.0 \mathrm{NS}$ & $4.5 b^{*}$ & $0.3 \mathrm{bB}$ & $0.9 \mathrm{~A}$ & $12.1 \mathrm{bNS}$ & $12.9 \mathrm{a}$ & Days & Yes & 14.8abNS & $15.7 \mathrm{ab}$ & & Days & No & Yes \\
\hline $\mathrm{C}$ & $3 \mathrm{~B}$ & $38 \mathrm{aA}$ & $3.0 \mathrm{~B}$ & $7.0 \mathrm{aA}$ & $0.6 \mathrm{abB}$ & $1.6 \mathrm{~A}$ & $12.0 \mathrm{bNS}$ & $10.4 \mathrm{~b}$ & 14 & 13.9 & 14.1bNS & $15.1 \mathrm{ab}$ & & 14 & $15.9 \mathrm{NS}$ & 16 \\
\hline $\mathrm{D}$ & $4 N S$ & $1 \mathrm{bc}$ & $4.0 \mathrm{NS}$ & $4.5 b^{*}$ & $0.6 \mathrm{abNS}$ & 1 & $11.1 \mathrm{bNS}$ & $11.4 \mathrm{ab}$ & 31 & 12.4 & $13.4 \mathrm{bNS}$ & $14.8 \mathrm{~b}$ & & 31 & $14.5 \mathrm{~B}$ & $15.8 \mathrm{~A}$ \\
\hline E & $1 \mathrm{nsB}$ & $0 \mathrm{c}$ & $3.5 \mathrm{NS}$ & $5.0 \mathrm{~b}$ & $0.5 \mathrm{bB}$ & $1.2 \mathrm{~A}$ & $12.2 \mathrm{bNS}$ & $12.4 \mathrm{ab}$ & 50 & 10.1 & 14.2abB & $16.8 \mathrm{aA}$ & & 50 & 13.0B & $15.5 \mathrm{~A}$ \\
\hline Control & $2 \mathrm{~B}$ & $37 a A$ & $3.5 \mathrm{~B}$ & $7.0 \mathrm{aA}$ & $0.6 \mathrm{abB}$ & $1.6 \mathrm{~A}$ & $11.5 \mathrm{bNS}$ & $12.5 \mathrm{abB}$ & Sign.r & $\mathrm{L}^{* * *}$ & $14.1 \mathrm{bB}$ & 16.3abA & Sign. & & $\mathrm{L}^{* * *}$ & NS \\
\hline \multicolumn{17}{|c|}{ With fertilizer } \\
\hline $\begin{array}{c}\text { All } \\
\text { gypsum }\end{array}$ & & & & $4.5 \mathrm{a}^{\mathrm{q}}$ & & $1.2 \mathrm{~ns}$ & & $11.9 \mathrm{~ns}$ & & & & $15.9 \mathrm{~ns}$ & & & & \\
\hline
\end{tabular}




\section{Agricultural Research \& Technology: Open Access Journal}

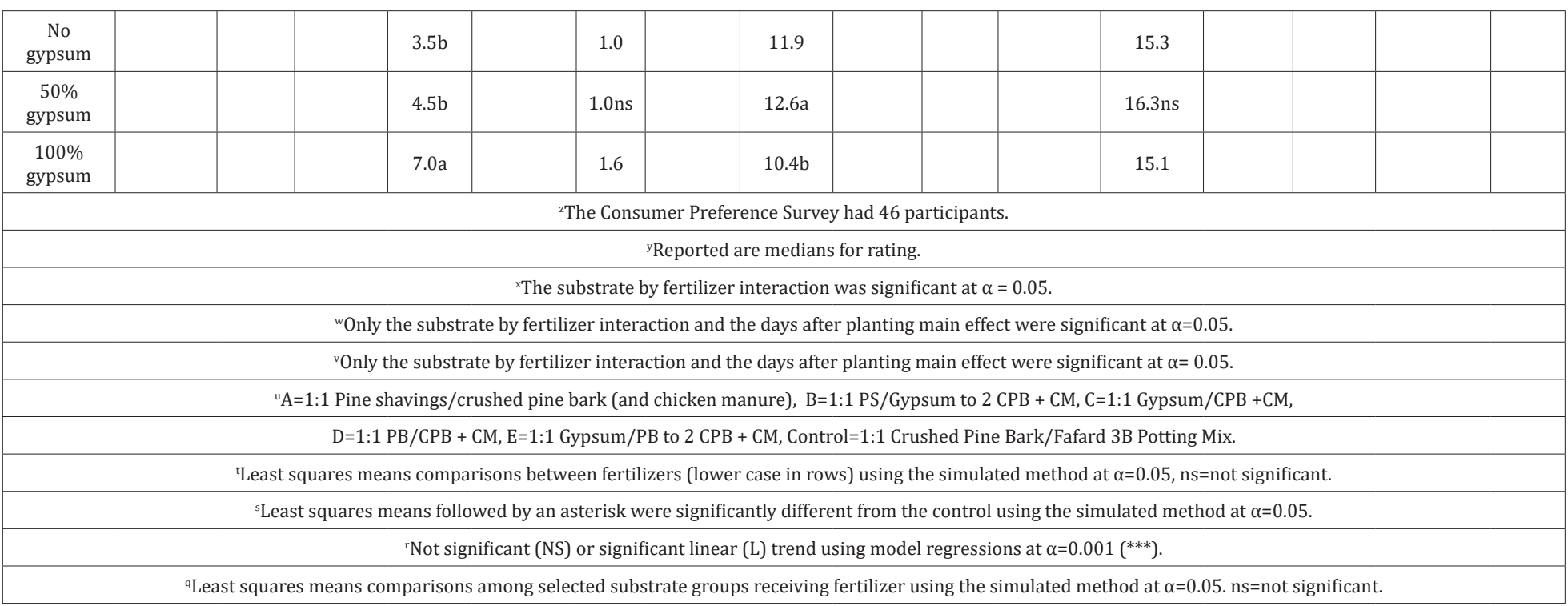

Table 4: Consumer preference and leachate readings at termination of Japanese Painted ferns grown in six substrates.

\begin{tabular}{|c|c|c|c|c|c|c|c|c|c|c|}
\hline & \multicolumn{2}{|c|}{$\begin{array}{c}\text { Consumer } \\
\text { Preferencez }\end{array}$} & \multicolumn{2}{|c|}{$\mathrm{pH}^{\mathrm{y}}$} & \multicolumn{2}{|c|}{ EC $^{\mathrm{x}}$} & \multicolumn{2}{|c|}{ Ca ppmw } & \multicolumn{2}{|c|}{$\mathbf{P ~ p p m}^{\mathbf{v}}$} \\
\hline & \multicolumn{2}{|c|}{ Fertilized } & \multicolumn{2}{|c|}{ Fertilized } & \multicolumn{2}{|c|}{ Fertilized } & \multicolumn{2}{|c|}{ Fertilized } & \multicolumn{2}{|c|}{ Fertilized } \\
\hline Substrate $^{u}$ & No & Yes & No & Yes & No & Yes & No & Yes & No & Yes \\
\hline A & $1 \mathrm{nsB}$ & $5 \mathrm{bAt}$ & $7.43 \mathrm{nsA}$ & 6.60abB & $0.42 \mathrm{bB}$ & $1.01 \mathrm{cA}^{*}$ & $45.2 \mathrm{bB} *$ & $106.3 \mathrm{bA}^{*}$ & $57.3 \mathrm{bB} *$ & $125.9 \mathrm{a} \mathrm{A}^{*}$ \\
\hline B & $1 \mathrm{nsB}$ & $1 \mathrm{bc}$ & $7.21 \mathrm{~A}$ & $6.96 \mathrm{aB}$ & $1.86 \mathrm{aB} * \mathrm{~s}$ & $2.45 \mathrm{aA}^{*}$ & $460.3 \mathrm{aB}^{*}$ & $639.7 \mathrm{bA} *$ & 10.7 dNS* $^{*}$ & $18.3 \mathrm{~b}$ \\
\hline C & 3B & $38 \mathrm{aA}$ & $7.16 \mathrm{NS}$ & $6.04 \mathrm{bc}$ & 2.01aNS* & $2.05 b^{*}$ & 691.8aNS* & $763.0 a^{*}$ & $19.3 \mathrm{cB}^{*}$ & $50.1 \mathrm{bA}$ \\
\hline $\mathrm{D}$ & $4 \mathrm{NS}$ & $1 \mathrm{bc}$ & $7.26 \mathrm{~A}$ & $6.52 \mathrm{abB}$ & $0.55 \mathrm{bB}$ & $1.24 \mathrm{cA}^{*}$ & $75.3 \mathrm{bNS} *$ & $147.8 \mathrm{~b}$ & $68.2 \mathrm{aB} *$ & $132.6 \mathrm{aA} *$ \\
\hline $\mathrm{E}$ & $1 \mathrm{nsB}$ & $0 \mathrm{c}$ & $7.16 \mathrm{~A}$ & $6.40 \mathrm{bB}$ & $2.08 \mathrm{aB}^{*}$ & $2.61 \mathrm{aA} *$ & 685.6aNS* & 736.4a* & $9.8 \mathrm{dNS} *$ & $25.4 \mathrm{~b}$ \\
\hline Control & $2 \mathrm{~B}$ & $37 a A$ & $7.09 \mathrm{~A}$ & $5.70 \mathrm{cB}$ & $0.21 \mathrm{bB}$ & $0.67 \mathrm{dA}$ & $14.9 \mathrm{cB}$ & $35.2 \mathrm{bA}$ & $1.7 \mathrm{eB}$ & $40.0 \mathrm{bA}$ \\
\hline \multicolumn{11}{|c|}{ With fertilizer } \\
\hline All gypsum & & & & $6.5 n s^{r}$ & & $2.4 \mathrm{a}$ & & $713.0 \mathrm{a}$ & & $31.3 \mathrm{~b}$ \\
\hline $\begin{array}{c}\text { No } \\
\text { gypsum }\end{array}$ & & & & 6.6 & & $1.1 \mathrm{~b}$ & & $127.1 \mathrm{~b}$ & & $129.3 a$ \\
\hline $\begin{array}{c}50 \% \\
\text { gypsum }\end{array}$ & & & & 6.7 & & $2.5 \mathrm{a}$ & & 688.0ns & & $21.9 \mathrm{~b}$ \\
\hline $\begin{array}{c}100 \% \\
\text { gypsum }\end{array}$ & & & & 6 & & $2.1 \mathrm{~b}$ & & 763 & & $50.1 \mathrm{a}$ \\
\hline
\end{tabular}

${ }^{2}$ The Consumer Preference Survey had 46 participants.

${ }^{\mathrm{Y}}$ The substrate by fertilizer interaction was significant at $\alpha=0.05$.

${ }^{x}$ The substrate by fertilizer interaction was significant at $\alpha=0.05$.

wThe substrate by fertilizer interaction was significant at $\alpha=0.05$.

vThe substrate by fertilizer interaction was significant at $\alpha=0.05$.

tLeast squares means comparisons among substrates (lower case in rows) using the simulated method at $\alpha=0.05$, ns=not significant.

${ }^{s}$ Least squares means followed by an asterisk were significantly different from the control using the simulated method at $\alpha=0.05$.

${ }^{\mathrm{r}}$ Least squares means comparisons among selected substrate groups receiving fertilizer using the simulated method at $\alpha=0.05$. $n=$ not significant. 
Gerbera daisy plants that received the most "yes" votes were those that received fertilizer, which was true for the other species (Tables 5-6). However, different from the ferns, treatment " $\mathrm{C}$ " (100\% gypsum) received no votes. The top three were Treatments "B", "D" and "F" (control). The top performing treatment was the control that had no gypsum (received 41 out of 108 votes), the second was treatment "B" that contained $50 \%$ gypsum (originally) (received 31 out of 108 votes), and treatment "D" that was $100 \%$ pine bark (originally) (received 24 votes out of 108). Gerbera daisy performed best either without any gypsum, or only up to $25 \%$ (originally $50 \%$ gypsum from the broiler study) in the substrate. Each species had a potential of receiving 135 "yes" votes. Gerbera daisy received 108 "yes" votes in the survey, Australian sword ferns received 121, and Japanese Painted ferns received 94 "yes" votes.

Table 5: Consumer preferences and leachate readings at termination of Gerbera Daisies grown in six substrates.

\begin{tabular}{|c|c|c|c|c|c|c|c|c|c|c|c|c|c|c|c|c|}
\hline \multirow[b]{3}{*}{ Substratev } & \multirow{2}{*}{\multicolumn{2}{|c|}{$\begin{array}{c}\begin{array}{c}\text { Consumer } \\
\text { Preferencez }\end{array} \\
\text { Fertilized }\end{array}$}} & \multicolumn{6}{|c|}{ Heighty } & \multicolumn{6}{|c|}{ Sizex } & \multirow{2}{*}{\multicolumn{2}{|c|}{$\begin{array}{c}\text { Dry Weightw } \\
\text { Fertilized }\end{array}$}} \\
\hline & & & \multicolumn{2}{|c|}{ Fertilized } & & & & & \multicolumn{2}{|c|}{ Fertilized } & & & & & & \\
\hline & No & Yes & No & Yes & & & & & No & Yes & & & & & No & Yes \\
\hline A & 0nsB & $7 \mathrm{dAu}$ & 9.8abNS & $10.1 b^{*} \mathrm{t}$ & \multicolumn{4}{|c|}{ Fertilizer/Height } & 16.9abNS* & 17.3ab & \multicolumn{4}{|c|}{ Fertilizer/Size } & $2.3 \mathrm{abB} *$ & 4.5abA \\
\hline $\mathrm{B}$ & $1 \mathrm{~B}$ & $31 \mathrm{bA}$ & $10.4 \mathrm{aNA} *$ & $11.0 \mathrm{ab}$ & Days & & No & Yes & 18.0aNS* & 17.3ab & & Days & No & Yes & 3.1aNS* & $3.8 a b^{*}$ \\
\hline $\mathrm{C}$ & $2 \mathrm{NS}$ & $0 \mathrm{e}$ & 9.4abB & $11.1 \mathrm{abA}$ & & 14 & $11.5 \mathrm{NS}$ & 12.1 & $15.9 \mathrm{bcNS} *$ & $16.6 b^{*}$ & & 14 & $16.1 \mathrm{NS}$ & 15.5 & $1.4 \mathrm{bB} *$ & $3.0 \mathrm{bA}^{*}$ \\
\hline $\mathrm{D}$ & oB & $24 \mathrm{Ac}$ & 10.0abNS & $10.8 \mathrm{ab}$ & & 31 & $9.1 \mathrm{NS}$ & 9.6 & $16.8 \mathrm{abNS} *$ & 17.8ab & & 31 & $16.1 \mathrm{NS}$ & 16.9 & $2.6 \mathrm{aB}^{*}$ & 4.9abA \\
\hline E & ONS & $1 \mathrm{de}$ & $8.3 \mathrm{bB}$ & $10.0 \mathrm{bA}$ & & 50 & $7.7 \mathrm{~B}$ & $11.1 \mathrm{~A}$ & 14.9adB & 17.3abA & & 50 & $15.9 \mathrm{~B}$ & $20.4 \mathrm{~A}$ & $2.0 \mathrm{abB}^{*}$ & 4.1 abA \\
\hline Ctrl & $1 \mathrm{~B}$ & $41 \mathrm{aA}$ & $8.4 \mathrm{bB}$ & $12.7 \mathrm{aA}$ & Sign.s & & $\mathrm{L}^{* * *}$ & $\mathrm{Q}^{* * *}$ & $13.7 \mathrm{~dB}$ & $19.2 \mathrm{aA}$ & Sign. & & NS & $\mathrm{L}^{* * *}$ & $0.6 \mathrm{cB}$ & $6.6 \mathrm{aA}$ \\
\hline \multicolumn{17}{|c|}{ With fertilizer } \\
\hline All gypsum & & & & $10.7 \mathrm{nsr}$ & & & & & & 17.1ns & & & & & & $3.6 \mathrm{~ns}$ \\
\hline No gypsum & & & & 10.5 & & & & & & 17.6 & & & & & & 4.7 \\
\hline $\begin{array}{l}50 \% \\
\text { gypsum }\end{array}$ & & & & $10.5 \mathrm{~ns}$ & & & & & & $17.4 \mathrm{~ns}$ & & & & & & $4.0 \mathrm{~ns}$ \\
\hline $\begin{array}{c}100 \% \\
\text { gypsum }\end{array}$ & & & & 11.1 & & & & & & 16.6 & & & & & & 3 \\
\hline \multicolumn{17}{|c|}{${ }^{\mathrm{z} T h e}$ Consumer Preference Survey had 46 participants. } \\
\hline \multicolumn{17}{|c|}{${ }^{y}$ Only the substrate by fertilizer and fertilizer by days after planting interactions were significant at $\alpha=0.05$. } \\
\hline \multicolumn{17}{|c|}{${ }^{x}$ Only the substrate by fertilizer and fertilizer by days after planting interactions were significant at $\alpha=0.05$. } \\
\hline \multicolumn{17}{|c|}{ 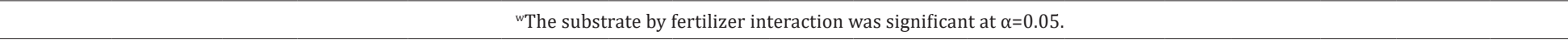 } \\
\hline \multicolumn{17}{|c|}{${ }^{\mathrm{v}} \mathrm{A}=1: 1$ Pine shavings/crushed pine bark (and chicken manure), $\mathrm{B}=1: 1 \mathrm{PS} /$ Gypsum to $2 \mathrm{CPB}+\mathrm{CM}, \mathrm{C}=1: 1 \mathrm{Gypsum} / \mathrm{CPB}+\mathrm{CM}$, } \\
\hline \multicolumn{17}{|c|}{$\mathrm{D}=1: 1 \mathrm{~PB} / \mathrm{CPB}+\mathrm{CM}, \mathrm{E}=1: 1$ Gypsum/PB to $2 \mathrm{CPB}+\mathrm{CM}$, Control=1:1 Crushed Pine Bark/Fafard 3B Potting Mix } \\
\hline \multicolumn{17}{|c|}{ "Least squares means comparisons between fertilizers (lower case in rows) using the simulated method at $\alpha=0.05$, ns=not significant. } \\
\hline \multicolumn{17}{|c|}{ tLeast squares means followed by an asterisk were significantly different from the control using the simulated method at $\alpha=0.05$. } \\
\hline \multicolumn{17}{|c|}{ sSignificant linear (L) or quadratic (Q) trends using model regressions at $\alpha=0.001\left({ }^{* * *}\right)$. } \\
\hline
\end{tabular}

Table 6: Consumer preferences and leachate readings of Grbera Daisies at termination in six substrates.

\begin{tabular}{|c|c|c|c|c|c|c|}
\hline & \multicolumn{2}{|c|}{ Consumer Preference ${ }^{z}$} & \multicolumn{2}{|c|}{ Ratingy } & \multicolumn{2}{|c|}{$\operatorname{Spad}^{\mathrm{x}}$} \\
\hline & \multicolumn{2}{|c|}{ Fertilized } & \multicolumn{2}{|c|}{ Fertilized } & \multicolumn{2}{|c|}{ Fertilized } \\
\hline Substratew & No & Yes & No & Yes & No & Yes \\
\hline A & 0nsB & $7 \mathrm{dAv}$ & $4.0 \mathrm{aNS}$ & $2.0 \mathrm{~b}^{*}$ & $29.6 \mathrm{bcB}$ & $36.2 \mathrm{bcA}^{*}$ \\
\hline B & $1 \mathrm{~B}$ & $31 \mathrm{bA}$ & $4.5 \mathrm{aNS} * \mathrm{u}$ & $2.5 b^{*}$ & 33.9abNS* & $38.7 \mathrm{bc} *$ \\
\hline $\mathrm{C}$ & $2 \mathrm{NS}$ & $0 \mathrm{e}$ & $1.0 \mathrm{bB}$ & $3.5 \mathrm{abA}$ & $29.9 \mathrm{bcB} *$ & 43.5abA \\
\hline $\mathrm{D}$ & OB & $24 \mathrm{Ac}$ & $2.0 \mathrm{bB}$ & $5.0 \mathrm{aA}$ & $36.5 \mathrm{aNS}^{*}$ & $40.7 \mathrm{abc}$ \\
\hline $\mathrm{E}$ & ONS & $1 \mathrm{de}$ & $2.0 \mathrm{bNS}$ & $2.0 \mathrm{~b}^{*}$ & 30.3abcNS & $33.5 c^{*}$ \\
\hline Ctrl & 1B & $41 \mathrm{aA}$ & $1.0 \mathrm{bB}$ & $6.0 \mathrm{aA}$ & $26.6 \mathrm{cB}$ & $47.9 \mathrm{aA}$ \\
\hline \multicolumn{7}{|c|}{ With fertilizer } \\
\hline All gypsum & & & & 3.0nst & & $38.6 \mathrm{~ns}$ \\
\hline No gypsum & & & & 4 & & 38.5 \\
\hline
\end{tabular}


Agricultural Research \& Technology: Open Access Journal

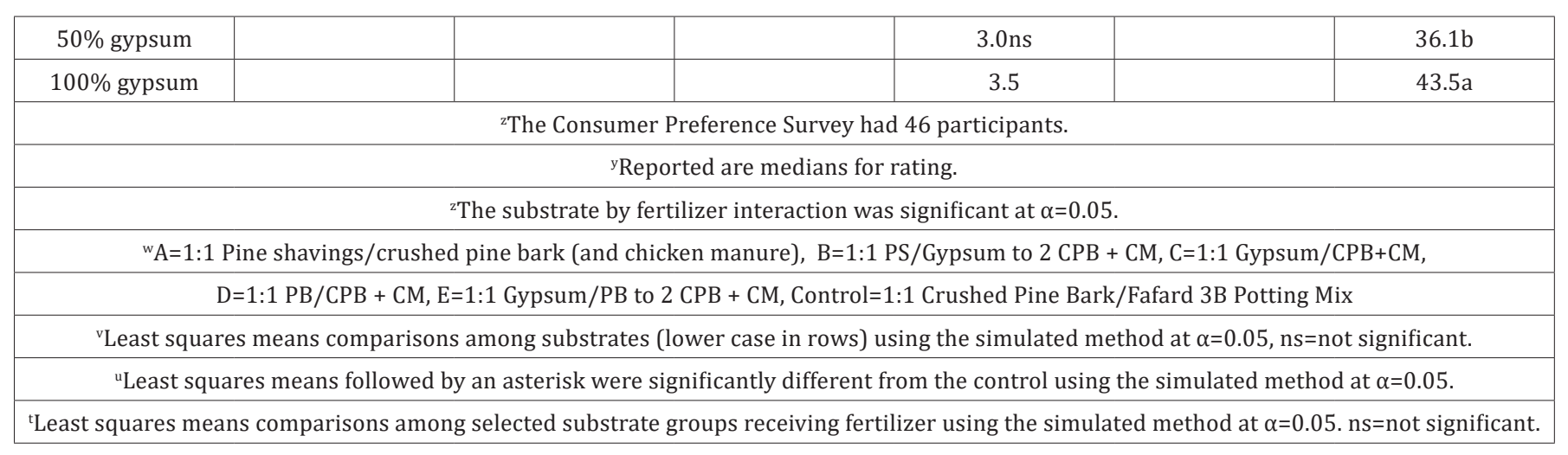

\section{Plant dry weight}

In all three species, fertilizer and substrate determined plant dry weights, and gypsum was not important. For Japanese painted ferns (Table 3) and Gerbera daisy (Table 5), the interaction between substrate and fertilizer was significant, but not for Australian sword ferns. The substrate and the fertilizer were the significant main effects on dry weight for Australian sword ferns (Table 1).

\section{Australian sword ferns}

The interaction between substrate and fertilizer was not significant, but both were significant main effects. The least squares mean of plants not fertilized was $3.0 \mathrm{~g}$ and those fertilized was $5.4 \mathrm{~g}$. Substrates with the highest dry weights were "A"- 4.0g (1:1 PS:CPB), "B"- 4.5g (1:1:2 PS:G:CPB), and “D"- 5.3g (1:1 PB:CPB) and were statistically the same. However, the other three treatments "C" (1:1FGDG:CPB), "E" (1:1:2FGDG:PB:CPB) and the control (1:1 PB: Fafard 3B) were not far behind (3.4 to $3.7 \mathrm{~g}$ ) and were statistically the same, which included the control (Table 1).

\section{Japanese painted ferns}

The interaction between substrate and fertilizer was significant. Substrates "B" (1:1:2 FGDG:PS:CPB), “C" (1:1FGDG:CPB), "E"(1:1:2 FGDG:PB:CPB) and the control (1:1 CPB:Fafard3B) had an increase in dry weight with fertilizer but were statistically the same ( 0.9 to $1.6 \mathrm{~g})$. Substrate " $\mathrm{C}$ " and the control were both $1.6 \mathrm{~g}$. Interestingly, substrate A (1:1 PS:CPB) had a greater dry weight without fertilizer and the substrate E did not have a significant increase with fertilizer (Table 3).

\section{Gerbera daisies}

There was a significant interaction between substrate and fertilizer and no significant benefit from gypsum. Plants in all treatments increased in dry weight from receiving fertilizer. Dry weights for all treatments except " $\mathrm{C}$ " were statistically the same, ranging from 3.8 to $4.9 \mathrm{~g}$ with the control at $6.6 \mathrm{~g}$. Treatment " $\mathrm{C}$ " had slightly less dry weight of 3.0g. Therefore, the biggest gain was in the control and the least in " $\mathrm{C}$ " (Table 5).

All species and treatments performed best with fertilizer with one exception; where Japanese painted ferns, showed no increase in dry weight with fertilizer for treatment " $\mathrm{A}$ " 1 : 1
PS:CPB). Also, for all three species gypsum had no significant effect on dry weight of plants that received fertilizer. The two species that had an interaction between substrate and fertilizer were Gerbera daisy and Japanese painted ferns, which suggest that plants receiving fertilizer, regardless of which substrates were used would more increase in dry weight.

\section{Size (Table 1,3\&5)}

\section{Australian sword ferns}

There were no significant interactions for plant size. Overall, all plants receiving fertilizer were significantly larger than those not receiving fertilizer on each day after transplanting that measurements were taken (Table 1).

\section{Japanese painted ferns}

Interactions between substrate and fertilizer at each measurement date were significant. All substrates were statistically the same with fertilizer except for " $D$ " (1:1 PB:CPB) which was a bit smaller. Without fertilizer, substrates "A", "B", and "E" were statistically the same, and "C", "D", and "F" (control) were the same. For substrates " $\mathrm{A}$ ", "B", "C", and " $\mathrm{D}$ " the difference in size between those fertilized and those that were not was not significant. Only substrates "E" and control were significantly different, and those fertilized were significantly larger in size (Table 3).

\section{Gerbera daisy}

Interaction between substrate and fertilizer was significant as well as the interaction between fertilizer and date of measurement. Overall, plants that were not fertilized had no significant change in size, but those that were fertilized had significant increases in size progressively and consistently from day 14 to day 50 . Similar to the Japanese painted ferns, substrates "A", "B", "C", and "D" with and without fertilizer were not different. However, like the Japanese painted ferns, substrates " $E$ " and control that were fertilized were significantly larger than those that were not fertilized (Table 5).

\section{SPAD}

Both Australian sword ferns and Gerbera daisy had significant interactions between substrate and fertilizer, and SPAD Values for Gerbera daisy were also different between substrates that had $25 \%$ vs. $50 \%$ gypsum. 


\section{Australian sword ferns}

In general, SPAD readings increased over time. The difference between Day 31 (38.7) and Day 50(42.1) was significantly higher. The interactions between substrate and fertilizer indicate that all substrates increased in chlorophyll content with fertilization. Substrates "A", "B", "D" and "E" without fertilizer were significantly different from the control (in this case, the control had a much lower reading. Substrate C (50\% gypsum) and the control increased with fertilizer (almost double). The differences between substrates that had $25 \%, 50 \%$ or no gypsum were not statistically different (Table 1 ).

\section{Gerbera daisy}

Interactions between substrate and fertilizer were significant and there were significant differences between substrates with $25 \%$ and $50 \%$ gypsum (Table 5). Substrates "A" (1:1 PS: CPB), C and the control were significantly higher with fertilizer, however, the SPAD readings for substrates " $B$ " (1:1:2 G:PS:CPB), "D" and "E" were not significantly different or increased with fertilizer. The substrates with $50 \%$ gypsum were not significantly different from those with no gypsum, however, the difference between $25 \%$ gypsum (36.1) vs. $50 \%$ gypsum (43.5) was significant and higher with more gypsum.

\section{pH}

All three species of plants had a significant interaction between substrate and fertilizer in regards to $\mathrm{pH}$. With only one exception, all substrates for all species had a higher than recommended $\mathrm{pH}$ without fertilizer, including the industry standard control. The one exception was the substrate "C" $(1: 1$ FGDG: $\mathrm{CPB}$ ) for the Australian sword ferns that was within BMP range both with and without fertilizer (without fertilizer: pH of 6.56 and with fertilizer: pH of 6.49) (SNA, 2013). The pH decreased in all plants for all substrates and all species that were fertilized. The only substrate that was consistently within the recommended $\mathrm{pH}$ range for all species was the control (1:1 PB: Fafard 3B) only when it received fertilizer. Without fertilizer, the control was above recommended range, the same as the other substrates.

\section{Australian sword fern}

$\mathrm{Ph}$ for three substrates fell into recommended levels after fertilizing: "C" (1:1FGDG: CPB 6.49), "D" (1:1 PB: CPB 6.02), and control (1:1CPB:Fafard3B 5.63). The data indicated two of the substrates with gypsum (that received fertilizer) were both statistically significant (Table 2).

\section{Japanese painted ferns}

Among fertilized substrates, there was no statistical difference with or without gypsum (of any amount) (Table 4).

\section{Gerbera daisy}

Only the control substrate with fertilizer decreased in $\mathrm{pH}$ to an acceptable recommended level (5.63). All other fertilized substrates ranged between 6.66 to $7.27 \mathrm{pH}$ (Table 7).

Table 7: Consumer preferences and leachate readings at termination of Gerbera Daisies grown in six substrates.

\begin{tabular}{|c|c|c|c|c|c|c|c|c|c|}
\hline & \multicolumn{2}{|c|}{ Consumer Preference ${ }^{z}$} & \multicolumn{2}{|c|}{$\mathrm{pH}^{\mathrm{y}}$} & \multicolumn{2}{|c|}{$\mathbf{E C}^{\mathbf{x}}$} & \multirow[t]{2}{*}{ Ca ppmw } & \multicolumn{2}{|c|}{ P ppmv } \\
\hline & Fertilized & & Fertilized & & Fertilized & & & Fertilized & \\
\hline Substrateu & No & Yes & No & Yes & No & Yes & & No & Yes \\
\hline A & OnsB & $7 \mathrm{dAt}$ & 7.75aA*s & $6.75 b B^{*}$ & $0.39 \mathrm{aB}^{*}$ & $1.00 \mathrm{bA}$ & $74.72 \mathrm{bc}$ & $51.27 a^{*}$ & $131.70 a^{*}$ \\
\hline $\mathrm{B}$ & $1 \mathrm{~B}$ & $31 \mathrm{bA}$ & $7.36 \mathrm{bcNS} *$ & $7.27 \mathrm{a}^{*}$ & $2.05 \mathrm{abB}^{*}$ & $2.36 \mathrm{aA}^{*}$ & $637.64 a^{*}$ & $12.28 b^{*}$ & $12.82 \mathrm{~d}^{*}$ \\
\hline $\mathrm{C}$ & $2 \mathrm{NS}$ & $0 \mathrm{e}$ & $7.03 \mathrm{cNS}$ & $6.78 b^{*}$ & $1.92 \mathrm{bB}^{*}$ & $2.33 \mathrm{a} A^{*}$ & $652.42 \mathrm{a}^{*}$ & $15.68 b^{*}$ & $40.90 c$ \\
\hline $\mathrm{D}$ & OB & $24 \mathrm{Ac}$ & 7.38abcA* & $6.66 \mathrm{bB} *$ & $0.40 \mathrm{cB}^{*}$ & $1.03 \mathrm{bA} *$ & $91.38 b^{*}$ & $55.74 a^{*}$ & $115.48 a b^{*}$ \\
\hline $\mathrm{E}$ & ONS & $1 \mathrm{de}$ & 7.41abA* & 7.17aB* & 2.08aNS* & $2.20 \mathrm{a}^{*}$ & $686.26 a^{*}$ & $10.00 b^{*}$ & $10.71 d^{*}$ \\
\hline Control & 1B & $41 \mathrm{aA}$ & $6.81 \mathrm{cA}$ & $5.63 \mathrm{cB}$ & $0.19 \mathrm{~dB}$ & $0.61 \mathrm{bA}$ & $34.11 \mathrm{c}$ & $0.49 c$ & $71.54 \mathrm{bc}$ \\
\hline \multicolumn{10}{|c|}{ With fertilizer } \\
\hline All gypsum & & & & 7.07ar & & $2.30 \mathrm{a}$ & $658.8 \mathrm{a}$ & & $21.5 b$ \\
\hline No gypsum & & & & $6.71 \mathrm{~b}$ & & $1.01 \mathrm{~b}$ & $83.1 \mathrm{~b}$ & & $123.6 \mathrm{a}$ \\
\hline $50 \%$ gypsum & & & & $7.22 \mathrm{a}$ & & $2.28 \mathrm{~ns}$ & $661.2 \mathrm{~ns}$ & & $11.8 \mathrm{~b}$ \\
\hline $\begin{array}{c}100 \% \\
\text { gypsum }\end{array}$ & & & & $6.78 b$ & & 2.3 & 652.4 & & $40.9 a$ \\
\hline \multicolumn{10}{|c|}{${ }^{2}$ The Consumer Preference Survey had 46 participants. } \\
\hline \multicolumn{10}{|c|}{${ }^{\mathrm{y}}$ The substrate by fertilizer interaction was significant at $\alpha=0.05$. } \\
\hline \multicolumn{10}{|c|}{ xThe substrate by fertilizer interaction was significant at $\alpha=0.05$. } \\
\hline \multicolumn{10}{|c|}{${ }^{\text {w}}$ The substrate by fertilizer interaction was significant at $\alpha=0.05$. } \\
\hline \multicolumn{10}{|c|}{ vThe substrate by fertilizer interaction was significant at $\alpha=0.05$. } \\
\hline
\end{tabular}


$\mathrm{uA}=1: 1$ Pine shavings/crushed pine bark (and chicken manure), B=1:1 PS/Gypsum to $2 \mathrm{CPB}+\mathrm{CM}, \mathrm{C}=1: 1 \mathrm{Gypsum} / \mathrm{CPB}+\mathrm{CM}$, $\mathrm{D}=1: 1 \mathrm{~PB} / \mathrm{CPB}+\mathrm{CM}, \mathrm{E}=1: 1$ Gypsum/PB to $2 \mathrm{CPB}+\mathrm{CM}$, Control=1:1 Crushed Pine Bark/Fafard 3B Potting Mix

tLeast squares means comparisons among substrates (lower case in rows) using the simulated method at $\alpha=0.05$, ns=not significant.

${ }^{\mathrm{s}}$ Least squares means followed by an asterisk were significantly different from the control using the simulated method at $\alpha=0.05$.

${ }^{\mathrm{r}}$ Least squares means comparisons among selected substrate groups receiving fertilizer using the simulated method at $\alpha=0.05$. ns=not significant.

Data for all substrates had a higher than recommended $\mathrm{pH}$ without fertilizer and all substrates had a decrease in $\mathrm{pH}$ where fertilizer was used. However, there was a wide range of results in the number of substrates that fall into an acceptable level (with fertilizer use) among the three species of plants used in this research. Gerbera daisy did not have any substrate except the control that fell into an acceptable level of $\mathrm{pH}$ (after fertilizing), but Australian sword ferns had three substrates that decreased to an acceptable range, and Japanese painted ferns had four.

\section{EC}

The sufficiency range for EC for ferns at 1.5-2.0mmhos/cm (per Casa Flora recommendations) is different from Gerbera daisy, at 1.2-1.5mmhos/cm (2:1 extraction method). For all three species of plants, there was a significant interaction between substrate and fertilizer, and data indicated that fertilizer increased the EC for all substrates within all three species. This increase was not a benefit; none of the fertilized plants were within recommended ranges and only one substrate (1:1:2 PS: FGDG: CPB) of the Japanese painted ferns was within range (1.86 $\mathrm{EC})$, and this was without fertilizer.

\section{Gerbera daisy}

Did not have one substrate (fertilizer or not) fall within the recommended range. The industry standard represented by the control had a $0.61 \mathrm{EC}$ reading and the rest had a 1.00 or close to 2.36 value (desired range: $1.2-1.5$ ). Interestingly, the three plants chosen as the best plants in the consumer survey were substrates with fertilizer: "B" (2.36), "D" (1.03) and "F" (control) (0.61) with three different EC levels, none of which were within BMP recommendations. Gypsum evaluations indicated that fertilized substrates without gypsum (1.01ECmmhos/cm) were closer to BMP recommendations than the substrates with gypsum (range of 2.28-2.33mmhos $/ \mathrm{cm}$ ).

\section{Australian sword ferns}

Have a recommended EC sufficiency range of 1.5-2.0mmhos/ $\mathrm{cm}$ (per Casa Flora). Here, there was a wide range of results from $0.21 \mathrm{mmhos} / \mathrm{cm}$ without fertilizer in the control representing the industry standard, with an increase to $0.79 \mathrm{mmhos} / \mathrm{cm}$ with fertilizer, to $2.54 \mathrm{mmhos} / \mathrm{cm}$ for substrate "E" (1:1:2 FGDG: PB: $\mathrm{CPB})$ with fertilizer. The closest to BMP values were substrates "B" (1:1:2 FGDG: PS: CPB) at 2.08mmhos/cm without fertilizer, and "C" (1:1 FGDG: CPB) both without $(2.08 \mathrm{mmhos} / \mathrm{cm})$ and with fertilizer $(2.14 \mathrm{mmhos} / \mathrm{cm})$, which is very close to their commended range $(1.5-2.0 \mathrm{mmhos} / \mathrm{cm}$ ) (Table 2). Substrate "C" (1:1 FGDG: CPB) also was one of the top two Australian sword fern plants chosen in the consumer preference survey.

\section{Japanese painted ferns}

Had almost the exact EC value results as the Australian sword ferns. Substrate "B" (1:1:2 G:PS:CPB) without fertilizer was within range $(1.86 \mathrm{mmhos} / \mathrm{cm})$, and substrate "C" (1:1 FGDG:CPB) with $(2.05 \mathrm{mmhos} / \mathrm{cm})$ and without fertilizer $(2.01 \mathrm{mmhos} / \mathrm{cm})$ were both close to the preferred BMP EC range $(1.5-2.0 \mathrm{mmhos} / \mathrm{cm})$ (Table 4). Here too, besides, the control, substrate "C" was one of the two top Japanese painted ferns to receive "Yes" votes in the Consumer Preference Survey as did substrate " $C$ " for the Australian sword ferns).

\section{Calcium (Ca)}

For all three species, there was a significant difference between substrates with no gypsum compared to the substrates that had some gypsum. For the Australian sword ferns and Gerbera daisy, the substrate was a significant main effect, but for the Japanese painted ferns, the interaction between the substrate and fertilizer was statistically significant.

\section{Australian sword ferns}

The substrate was a main effect. Substrates that contained gypsum had the highest Ca ("B", "C" and "E") and were statistically the same with a range of 564-623ppm. Calcium levels in substrates " $\mathrm{A}$ " and "D" were statistically the same (82-105ppm) and the control was the lowest at $28.2 \mathrm{ppm}$. The difference between substrates with $25 \%$ vs. $50 \%$ gypsum was not significant, but the substrates with any amount of gypsum had the highest amount of Ca (589.6ppm) vs. substrates without any gypsum averaged $28.2 \mathrm{ppm}$. Interestingly, one of the top substrates in the Consumer Preference Survey for Australian sword ferns was substrate "C" which originated as $100 \%$ gypsum This suggests that any amount of gypsum in the substrate would benefit this species. It also demonstrates that substrate " $\mathrm{C}$ " with $50 \%$ gypsum would be a viable alternative to one of the industry standards, which is substrate " $\mathrm{D}$ ", which consists of all pine bark (plus PL) (Table 2).

\section{Japanese painted ferns}

The interaction between substrate and fertilizer was statistically significant. Ca levels in "A", "B" and "F" were statistically the same and were significantly increased by fertilizer. Ca levels in substrates "C", "D" and "E" were not significantly increased with fertilizer. The results between the two substrates with $25 \%$ gypsum vs. $50 \%$ gypsum were not significant. However, the difference between the substrates that had some gypsum vs. none was significant. The substrates that had gypsum averaged 713ppm and those without any gypsum 
averaged 127.1ppm. Substrate "C" had 763.0ppm with fertilizer, but the control only had $35.2 \mathrm{ppm}$ with fertilizer. The ideal range is $10-15 \mathrm{ppm}$; they both meet that minimum criterion (Table 4).

\section{Gerbera daisy}

Substrate was a main effect, and the difference between substrates with some gypsum or none was significant as well. Ca levels in substrates "B", "C" and "E" were statistically the same, having a range of $652-686 \mathrm{ppm}$. Substrates with no gypsum ranged from $34-91$ ppmCa. The results between the two substrates with $25 \%$ gypsum $(661 \mathrm{ppm})$ vs. $50 \%$ gypsum (652ppm) were not different (Table 7).

\section{Phosphorus (P)}

All three species had a statistically significant interaction between substrate and fertilizer. All three species had increased $\mathrm{P}$ with fertilizer, but for all three, the $\mathrm{P}$ increases for substrates "B" and "E" were not significant. In addition, $P$ in all three species was different between treatments containing gypsum and those with no gypsum. P was also different between the two substrates with $25 \%$ vs. $50 \%$ gypsum.

\section{Australian sword ferns}

Substrate $\mathrm{x}$ fertilizer interactions was statistically significant with all substrates increasing in $\mathrm{P}$ with fertilizer, even though substrate $B$ and $E$ increases were not significant (Table 2). Also, substrates "A" and "E" with fertilizer were statistically the same and were the highest (109.4-135.8ppm), compared to the control with fertilizer (45.8ppm), "B" with fertilizer (14.1ppm) and "E" with fertilizer (29.6ppm). In the case of phosphorus, more is not always better (as in most nutrients) and when comparing no gypsum to some gypsum in the substrate, the gypsum reduced the amount of phosphorus could be a helpful benefit if trying to limit excess $\mathrm{P}$ runoff by binding with $\mathrm{Ca}$.

\section{Japanese painted ferns}

The substrate $\mathrm{x}$ fertilizer interactions was significant. Just like in the other two species, $\mathrm{P}$ in all substrates increased with fertilizer but the difference in substrate "B" and "E" were not significant. The results from treatments containing gypsum compared with no-gypsum and the two $25 \%$ gypsum treatments compared with $50 \%$ gypsum treatments were almost identical to those of the Australian sword ferns (Table 4). The statistically significant reading is much higher, again, indicating the usefulness of calcium in binding with excess phosphorus.

\section{Gerbera daisy}

The results for Gerbera dasies were similar to the other two species. The substrate $\mathrm{x}$ fertilizer interaction was statistically significant. $\mathrm{P}$ in all substrates increased with fertilizer with substrates "B" and "E" not significant. Leachates from substrates containing gypsum had the lowest amounts of $\mathrm{P}$ indicating the binding capacity of the Ca from gypsum. Substrates "A" and "D" which contained no gypsum had the highest $\mathrm{P}$ (Table 7).

\section{Final Discussion}

The Consumer Preference Survey was a useful tool in discerning the value of substrates as a potential alternative substrate. It should be noted that all top vote earners had been fertilized. For Australian sword ferns and Japanese painted ferns, the substrates with gypsum or those grown in the industry standard of $\mathrm{PB}$ :Fafard $3 \mathrm{~B}$ received the most votes. For Gerbera daisy, " $F$ " (the control) received the top votes (41), with the second level of votes (31) going to substrate "B" (25\% gypsum). However, surprisingly, substrate " $\mathrm{C}$ " (originating from $100 \%$ gypsum poultry bedding) did not receive any votes for Gerbera daisy. Therefore, from this, substrate "C" would not be recommended for Gerbera daisy, but could be used as an alternative substrate for both Australian sword ferns and Japanese painted ferns if the economics and availability worked out to the grower's advantage.

Phosphorus did not seem to correlate with success in the Consumer Preference Survey but substrate $\mathrm{x}$ fertilizer interaction proved significant in how much $\mathrm{P}$ was available. This is significant in light of the green industry's ever present concern about environmental impact and water quality. P increased in all substrates when fertilized, however, the greatest increase in $\mathrm{P}$ were the substrates with no gypsum, indicating that Cain the gypsum served to bind $\mathrm{P}$, thereby decreasing leachable P. However, not all $\mathrm{P}$ was bound, and all substrates retained sufficient $P$ for the needs of the plant. The evidence of the benefit of Ca can be seen in the Consumer Preference Survey as well where substrates with gypsum consistently received the most number of votes, along with the two substrates that are already industry standards (PB:Fafard 3B and 100\% PB).In addition, the substrates with gypsum flocculated, forming aggregates, which facilitated drainage. Poor or slow drainage was an issue for other substrates, and the growth and performance in these substrates was not as strong.

\section{References}

1. Lu W, Sibley JL, Gilliam CH, Bannon JS, Zhang Y (2006) Estimation of U.S. bark generation and implications for horticultural industries. J Environ Hort 24(1): 29-34.

2. Murphy AM (2011) Evaluation of sustainable alternative substrate components for container plant production. Auburn Univ, Auburn, USA.

3. Sibley JL, Gilliam CH, Foshee WG, Wright AN, Fain GB (2005) Development of nursery crop substrates from recycled materials and composted poultry litter. Proc Southern Nur Assn Res Conf 50:127-130.

4. Behe BK, Donald J, Gilliam CH (1991) Consumer evaluation of a soilless potting mix with composted broiler litter. Proc Southern Nur Assn Res Conf 36: 348-350.

5. Gilliam CH, Donald JO, Bice VC (1991) Methods for commercial utilization of composted broiler litter in the ornamental and landscape industries. Auburn University, Auburn, USA.

6. Marble SC (2009) Evaluation of composted poultry litter use in horticulture. Auburn Univ, Auburn, USA.

7. Pickens JM, Sibley JL, Gilliam CH, Bilgili SF, Hess JB, et al. (2009) Evaluation of bark-based poultry litter as a substrate component in nursery 
crop production. Proc SNA Res Conf 54: 121-123.

8. Chen L, Dick AW (2011) Gypsum as an agricultural amendment general use guidelines. Ohio State Univ Ext, USA.

9. Mayfield JL, Sibley JL, Simone EH, Eakes DJ (2002) Effect of liming materials on media $\mathrm{pH}$ and overall growth and nutrition of dwarf nandina. J Plant Nutr 25: 1377-1387.

10. Southern Nursery Association (2013) Best management practices: Guide for producing nursery crops. ( $3^{\text {rd }}$ edn), Southern Nur Assn, Atlanta, USA.

11. Thaxton BR (2011) Evaluation of manufactured organic soil amendments for consumer use. Auburn Univ, Auburn, USA.

12. Dawson CJ, Hilton J (2011) Fertliser availability in a resource-limited world: Production and recycling of nitrogen and phosphorus. Food Policy 36:14-22.

13. Gouin F (1980) Introduction to the symposium. Hort Science 15(2):161.

14. Ksheem AM, Mc Bennett JL, Antille DL, Raine SR (2015) Towards a method for optimized extraction of soluable nutrients from fresh and composted chicken manures. Waste Mgt 45: 76-90.

15. Raviv M, Lieth JH (2008) Soilless culture theory and practice. ( $1^{\text {st }}$ edn), Elsevier, London, UK.

16. Torbert HA, Watts DB (2014) Impact of flue gas desulfurization gypsum application on water quality in a coastal plain. J Environ Qual 43 273-280.

17. Agriculture and Agri-Food Canada (1994) Best management practices: Livestock and poultry waste management. Agriculture and Agri-food Canada, Ontario, Canada.

18. Nelson PV (2012) Greenhouse operation and management. ( $7^{\text {th }}$ edn), Prentice Hall, Englewood Cliffs, New Jersy, USA.

19. Brymer WJ (2008) Assessment of composting methods for use in the green industry. Auburn Univ, Auburn, USA.

20. Kelleher BP, Leahy JJ, Henihan AM, O’Dwyer TF, Sutton D, et al. (2002) Advanvces in poultry litter disposal technology - a review. Bioresource Tech 83(1): 27-36.

21. Ritter WF, Chirnside AEM, Scarborough RW (1986) Effect of agricultural activities on ground-water quality. ASAE Summer Meeting Paper, pp. 86-2025.
22. Wells DE (2011) Applications of poultry litter ash in greenhouse crop production. Louisiana State Univ, Baton Rouge, USA.

23. Wells DE (2008) Evaluation of spent tea grinds as an alternate horticultural substrate component. Auburn Univ, Auburn, USA.

24. Regulski FJ (1982) Evaluation of a gasifier residue as a container medium for woody or namentals. Hort Science17: 209-210.

25. USDA-ARS (2013) USDA-ARSflue gas desulfurization gypsum project. US Dept Agr, Washington, USA.

26. Giddens J, Barnett AP (1980) Soil loss and microbiological quality of runoff from land treated with poultry litter. J Environ Quality 9(3): 518520.

27. USDA (2007) Utility co-op connection Georgia alternative energy plant to be fueled by wood and poultry waste. USDA/Rural Development. US Dept Agr, Washington, USA.

28. Bilgili SF, Hess JB, Blake JP, Macklin KS, Saenmahayak B, et al. (2009) Influence of bedding material on foot pad dermatitis in broiler chickens. J Appl Poultry Res 18: 583-589.

29. Hess JB, Watts DB, Torbert HA, Sibley JL, Davis JD (2017) Gypsum as a bedding source for broiler chickens. SASS ASA-SR Branch Proceedings (Abstr.). Mobile, Auburn, USA.

30. Glomba M, Szmigielska E (2011) Conversion of calcium carbonate into synthetic gypsumin flue gas desulphurization technology using wet limestone method. Tech Trans Chem 108(8): 49-59.

31. Kessler JR (2002) Fertilizing greenhouse crops in Alabama. Alabama Coop Ext System ANR-1221.

32. Wright RD (1986) The pour-thru nutrient extraction procedure. Hort Science 21: 227-229.

33. Bilgili SF, Hess JB, Sibley JL, Torbert HA, Watts DB, et al. (2015) Gypsum as bedding for rearing broiler chickens. USDA-ARS Res Project \#429173.

34. Whitcomb CE (1984) Plant production in containers. Lacebark Publications, Stillwater, USA.

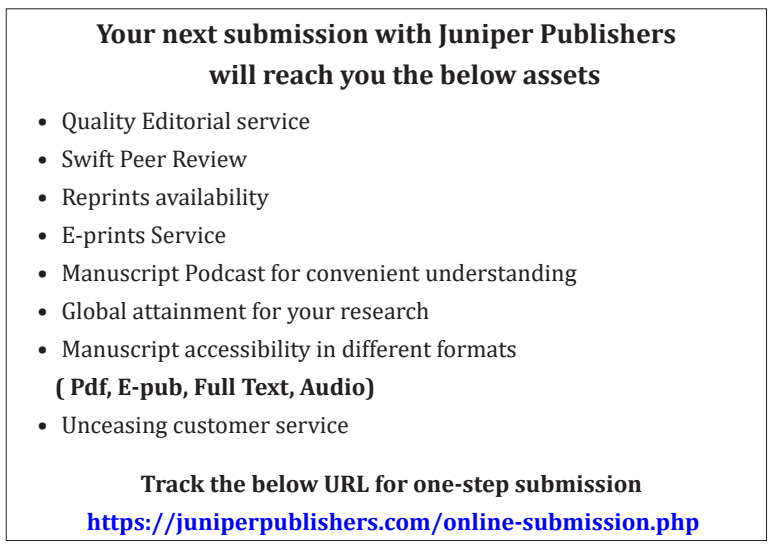

